\title{
Relativistic quantum motion of spin-0 particles under the influence of noninertial effects in the cosmic string spacetime
}

\author{
L. C. N. Santos ${ }^{\mathrm{a}}$, C. C. Barros Jr. \\ Depto de Física - CFM, Universidade Federal de Santa Catarina, CP. 476, Florianópolis, SC CEP 88040-900, Brazil
}

Received: 1 June 2017 / Accepted: 18 December 2017 / Published online: 10 January 2018

(C) The Author(s) 2018. This article is an open access publication

\begin{abstract}
We study solutions for the Klein-Gordon equation with vector and scalar potentials of the Coulomb types under the influence of noninertial effects in the cosmic string spacetime. We also investigate a quantum particle described by the Klein-Gordon oscillator in the background spacetime generated by a cosmic string. An important result obtained is that the noninertial effects restrict the physical region of the spacetime where the particle can be placed. In addition, we show that these potentials can form bound states for the Klein-Gordon equation in this kind of background.
\end{abstract}

\section{Introduction}

Quantum field theory (QFT) in curved spacetime can be considered as a first approximation to quantum gravity. Moreover, to make a consistent quantum field theory in a gravitational background, it is necessary to analyze the single particle states, in this way, efforts have been made in order to find an adequate formulation of the relativistic equation of motion for particles in a curved spacetime. In recent years, there has been a significant increase of interest in the study of gravitational effects on quantum mechanical systems (single particle states) [1-14]. In this context it has been shown that the energy spectrum associated with one-electron atoms in an arbitrary curved spacetime is different from the one obtained in the usual flat Minkowski spacetime [4]. The energy levels are shifted by the gravitational field and the effects of the curvature appear as perturbations in the relativistic fine structure. In addition, the physics of a neutrino in a curved metric is considered in [14] by Wheeler and Brill, who presented a detailed analysis of the interaction of neutrinos and gravitational fields.

Cosmic strings are another kind of system that may be investigated with this purpose. They are very interesting sys-

a e-mail: luis.santos@ufsc.br tems, which are supposed to be formed during a symmetry breaking phase transition in the early universe [15-21] and may be considered as topological defects in the spacetime structure predicted by a large class of theories. These structures are candidates for the generation of observable astrophysical phenomena such as high energy cosmic rays, gamma ray burst and gravitational waves [22]. The recent discovery of gravitational waves by the LIGO collaboration [23] suggests that a promising way to detect the cosmic strings is to search for the gravitational-wave radiation they would produce.

A key feature is that the spacetime around them is locally flat, but this is not a global propriety. The presence of such kind of topological defects can also influence the behavior of particles of a quantum system, for example, in [9] the Dirac equation has been solved in the presence of a Coulomb and a scalar potentials in the cosmic string spacetime and it was shown that its presence destroys the degeneracy of all the energy levels. In a recent work [24], solutions of the KleinGordon equation in Gödel-type spaces with an embedded cosmic string are considered, and it was shown that the presence of cosmic strings in the spacetime breaks the degeneracy of energy levels in Som-Raychaudhuri, spherical and hyperbolic $\mathrm{G}$ ödel spaces. In [25], the effects of magnetic fields in the metric have been considered. A relativistic wave equation for spin 1/2 particles in the Melvin spacetime, a spacetime where the metric is determined by a magnetic field, has been obtained and the effects of very intense magnetic fields in the energy levels, as intense as the ones expected to be produced in ultra-relativistic heavy-ion collisions, has been investigated.

Noninertial effects on physical systems are another kind of aspect that have been studied in much work in the literature [26-34]. A special case of a noninertial system is the rotating frame of reference. In [35,36], a rotating frame in the Minkowski spacetime, is investigate. Notably, in those papers it was shown that the geometry of the spacetime can 
play the role of a hard-wall potential. Another example of a noninertial system is the Mashhoon effect, that is, the coupling of the spin of the particles with the angular velocity of the rotating frames and it arises from the influence of these noninertial frames when interference effects are considered [37].

In this contribution, we will study scalar bosons in a cosmic string spacetime by considering the Klein-Gordon equation with a vector potential $v(r)=\kappa / r$ and a scalar potential $s(r)=\eta / r$, where $r$ is the radial coordinate with $\eta$ and $\kappa$ constants. Afterwards, we will examine a similar problem, the Klein-Gordon oscillator inside a cosmic string spacetime. Moreover, a rotating frame in the conical spacetime will be considered in both cases, and we will show that noninertial effects restrict the physical region of the spacetime where the particle can be placed, and furthermore the energy levels are shifted by the noninertial effects on the particle. This interesting feature is an indicator of a nontrivial phenomenon: the coupling between the angular quantum number and the angular velocity of the rotating frame. Afterwards, we will show that these potentials can form bound states for the KleinGordon equation in this spacetime. This paper is part of a study in which we systematically explore the properties of quantum systems inside spaces with different kinds of structures [25,38].

The paper is organized as follows: In Sect. 2, we will describe the cosmic string spacetime and the transformation from spacetime coordinates to rotating coordinates. In Sect. 3, the Klein-Gordon equation with vector and scalar potentials of the Coulomb types in the cosmic string spacetime will be determined and in Sect. 4 the Klein-Gordon oscillator will be solved. Finally, Sect. 5 presents our conclusions. In this work, we use natural units where $c=G=$ $\hbar=1$.

\section{The cosmic string and the noninertial reference frame}

In this section we will describe the relationship between the metric of a cosmic string and the effects of the rotation of a reference frame. The cosmic string spacetime is a solution of Einstein's field equations and it describes a spacetime determined by an infinitely long straight string. The string spacetime is assumed to be static and cylindrically symmetric, and then the distance element representing this system can be written in the form $[2,19]$

$\mathrm{d} s^{2}=-\mathrm{d} t^{\prime 2}+\mathrm{d} r^{\prime 2}+\alpha^{2} r^{\prime 2} \mathrm{~d} \phi^{\prime 2}+\mathrm{d} z^{\prime 2}$,

where $\alpha=1-4 G \mu$ and $\mu$ is the mass density of the string. In this spacetime, the coordinate range is represented in the following way: the azimuthal angle range is $\phi^{\prime} \in[0,2 \pi)$ while $r^{\prime}$ and $z^{\prime}$ are $r^{\prime} \in[0, \infty)$ and $z^{\prime} \in(-\infty, \infty)$, respectively. The parameter $\alpha$ is related to the curvature of spacetime. It may assume values in which $\alpha \leq 1$ or $\alpha>1$, and in this case, it corresponds to a cosmic string spacetime with negative curvature. In this work, we are interested in studying the case $0<\alpha<1$. The transformation of the metric (1) for a rotating reference frame may be made by considering a coordinate transformation $[39,40]$

$t^{\prime}=t, \quad r^{\prime}=r, \quad \phi^{\prime}=\phi+\omega t, \quad z^{\prime}=z$,

where $\omega$ is angular velocity of the rotating frame, which we assume to be positive. Inserting this transformation into Eq. (1) we obtain the line element

$$
\begin{aligned}
\mathrm{d} s^{2}= & -\left(1-\alpha^{2} r^{2} \omega^{2}\right) \mathrm{d} t^{2}+2 \alpha^{2} r^{2} \omega \mathrm{d} t \mathrm{~d} \phi \\
& +\mathrm{d} r^{2}+\alpha^{2} r^{2} \mathrm{~d} \phi^{2}+\mathrm{d} z^{2}
\end{aligned}
$$

which may be associated with the covariant metric tensor

$$
g_{\mu \eta}=\left(\begin{array}{llll}
-\left(1-\alpha^{2} r^{2} \omega^{2}\right) & 0 & 0 & \alpha^{2} r^{2} \omega \\
0 & 1 & 0 & 0 \\
0 & 0 & 1 & 0 \\
\alpha^{2} r^{2} \omega & 0 & 0 & \alpha^{2} r^{2}
\end{array}\right)
$$

We can see that $g_{\mu \eta}$ is a non-diagonal metric tensor where the effects of the topology and the rotation of the reference frame are taken into account. An interesting feature of Eq. (3) is the condition

$0<r<1 / \alpha \omega$.

That condition is related to the fact that for $r>1 / \alpha \omega$ the velocity of the particle is greater than the velocity of the light, for this reason, it is convenient to restrict $r$ to the range $(0,1 / \alpha \omega)$. In this way, the wave function of the particle must vanish at $r=1 / \alpha \omega$ and consequently this system presents two different classes of solutions that depend on the value of the product $\alpha \omega$. The first case is obtained by adopting the limit $\alpha \omega \ll 1(1 / \alpha \omega \rightarrow \infty)$, which provides an analytical solution to the Klein-Gordon equation and as a second case, an arbitrary relation $\alpha \omega$ can be considered.

\section{Klein-Gordon equation with vector and scalar potentials of the Coulomb types in the cosmic string spacetime}

The Dirac equation is a wave equation that represents very well spin-1/2 particles in Minkowski spacetime. The spin-0 particles are represented by the usual Klein-Gordon equation which can be generalized to the curved spacetime case. In order to determine the generalization of the wave equation one may replace the ordinary derivatives by covariant derivative [38] in the Klein-Gordon equation in Minkowski spacetime, the result is 


$$
-\frac{1}{\sqrt{-g}} D_{\mu}\left(g^{\mu \nu} \sqrt{-g} D_{\nu} \psi\right)+m^{2} \psi=0,
$$

that is, the Klein-Gordon equation in a curved spacetime [41], where $m$ is the particle mass, $D_{\mu}=\partial_{\mu}-i e A_{\mu}$, and $e$ is the electric charge. A scalar potential $V(r)$ may be taken into account by making a modification on the mass term: $m \rightarrow m+V(r)$. Substituting this mass term into (6) we obtain the following differential equation:

$$
-\frac{1}{\sqrt{-g}} D_{\mu}\left(g^{\mu \nu} \sqrt{-g} D_{\nu} \psi\right)+(m+V)^{2} \psi=0 .
$$

This differential equation takes into account a scalar potential $V$ and a potential vector $A_{\mu}[42,43]$. In the following, we will obtain two classes of solutions of Eq. (7). First, we will consider the slow rotation regime, and then we consider an arbitrary relation $\alpha \omega$ in the spacetime.

By considering the line element (3) and the potential vector $A_{0}$, we obtain the following differential equation:

$$
\begin{aligned}
& {\left[-\left(\frac{\partial}{\partial t}-i e A_{0}\right)^{2}+\frac{1}{r}\left(\frac{\partial}{\partial r}\right) r\left(\frac{\partial}{\partial r}\right)\right.} \\
& +\frac{\partial^{2}}{\partial z^{2}}+\left(\frac{1-\alpha^{2} r^{2} \omega^{2}}{\alpha^{2} r^{2}}\right) \frac{\partial^{2}}{\partial \phi^{2}} \\
& \left.+2 \omega\left(\frac{\partial}{\partial \phi}\right)\left(\frac{\partial}{\partial t}+i e A_{0}\right)-(m+V)^{2}\right] \psi=0,
\end{aligned}
$$

that is, the Klein-Gordon equation in the cosmic string spacetime. One can see that Eq. (8) is independent of $t, z$ and $\phi$, so it is reasonable to write the solution as

$$
\psi(t, r, z, \phi)=e^{-i \varepsilon t} e^{i l \phi} e^{i p_{z} z} R(r),
$$

where $l=0, \pm 1, \pm 2, \pm 3, \ldots$, and $\varepsilon$ can be interpreted as the energy of the particle, $p_{z}$ is the momentum. Substituting (9) into Eq. (8), and by considering $A_{0}=\kappa / r$, we obtain the radial differential equation

$$
\begin{aligned}
& {\left[\frac{\mathrm{d}^{2}}{\mathrm{~d} r^{2}}+\frac{1}{r} \frac{\mathrm{d}}{\mathrm{d} r}+\frac{e^{2} \kappa^{2}-l^{2} / \alpha^{2}}{r^{2}}-(m+V)^{2}\right.} \\
& \left.\frac{2 \varepsilon e \kappa+2 \omega e \kappa l}{r}+(\varepsilon+\omega l)^{2}-p_{z}^{2}\right] R(r)=0,
\end{aligned}
$$

where the parameter $\alpha$ represents the deficit angle of the spacetime and $\alpha=1$ corresponds to the Minkowski spacetime. In this paper, we are interested in studying the case $\alpha<1$.

In this stage, we consider a scalar potential of the type $V(r)=\eta / r$, where $\eta$ is a constant; thus, substituting this potential into Eq. (10), we obtain the following expression:

$$
\left[\frac{\mathrm{d}^{2}}{\mathrm{~d} r^{2}}+\frac{1}{r} \frac{\mathrm{d}}{\mathrm{d} r}-\frac{\beta^{2}}{r^{2}}-\frac{2 \gamma}{r}-\delta^{2}\right] R(r)=0,
$$

where

$\delta^{2}=m^{2}+p_{z}^{2}-(\varepsilon+\omega l)^{2}, \quad \beta=\sqrt{l^{2} / \alpha^{2}+\eta^{2}-e^{2} \kappa^{2}}$,

$\gamma=-\varepsilon e \kappa-\omega e \kappa l+m \eta$.

We assume the relation $e^{2} \kappa^{2}<\eta^{2}$ so that $\beta$ is a real number. Now, we will consider the transformation of the radial coordinate

$\rho=2 \delta r$,

and as a result Eq. (11) will take the form

$$
\left[\frac{\mathrm{d}^{2}}{\mathrm{~d} \rho^{2}}+\frac{1}{\rho} \frac{\mathrm{d} R}{\mathrm{~d} \rho}-\frac{\beta^{2}}{\rho^{2}}-\frac{\gamma}{\delta \rho}-\frac{1}{4}\right] R(\rho)=0 .
$$

Normalizable eigenfunctions may be obtained if we propose the solution

$$
R(\rho)=\rho^{\beta} e^{-\frac{\rho}{2}} F(\rho),
$$

then substituting $R(\rho)$ (15) into Eq. (14), we obtain the differential equation that can be associated with the radial equation

$$
\rho \frac{\mathrm{d}^{2} F}{\mathrm{~d} \rho^{2}}+(2 \beta+1-\rho) \frac{\mathrm{d} F}{\mathrm{~d} \rho}+\left(-\beta-\frac{\gamma}{\delta}-\frac{1}{2}\right) F=0 .
$$

This is the confluent hypergeometric equation, which is a second order linear homogeneous differential equation where two independent solutions can be obtained. The solution of Eq. (16), regular at $\rho=0$, is given by the confluent hypergeometric function, which is denoted by

$F(\rho)={ }_{1} F_{1}\left(\beta+\frac{\gamma}{\delta}+\frac{1}{2}, 2 \beta+1 ; \rho\right)$.

If we consider the limit $\alpha \omega \ll 1$, that is, the slow rotation regime, the boundary condition implies that the solution

${ }_{1} F_{1}\left(\beta+\frac{\gamma}{\delta}+\frac{1}{2}, 2 \beta+1 ; \frac{2 \delta}{\alpha \omega} \rightarrow \infty\right)$

must be finite as $\rho_{0}=1 / \alpha \omega \rightarrow \infty$. So, due to the asymptotic behavior of the hypergeometric function, it is necessary that the ${ }_{1} F_{1}$ function be a polynomial function of degree $N$ and the parameter $\beta+\frac{\gamma}{\delta}+\frac{1}{2}$ should be a negative integer. These conditions imply that

$\beta+\frac{\gamma}{\delta}+\frac{1}{2}=-N, \quad N=0,1,2, \ldots$,

and combining this equation and Eq. (12) we finally obtain the energy spectrum,

$$
\begin{aligned}
\varepsilon= & \frac{e \kappa m \eta}{\zeta+e^{2} \kappa^{2}} \\
& \pm \sqrt{\zeta \frac{\left[\left(p_{z}^{2}+m^{2}\right)\left(\zeta+e^{2} \kappa^{2}\right)-m^{2} \eta^{2}\right]}{\left(\zeta+e^{2} \kappa^{2}\right)^{2}}}-\omega|l|
\end{aligned}
$$

where $\zeta=\left(N+\frac{1}{2}+\sqrt{l^{2} / \alpha^{2}+\eta^{2}-e^{2} \kappa^{2}}\right)^{2}$. 


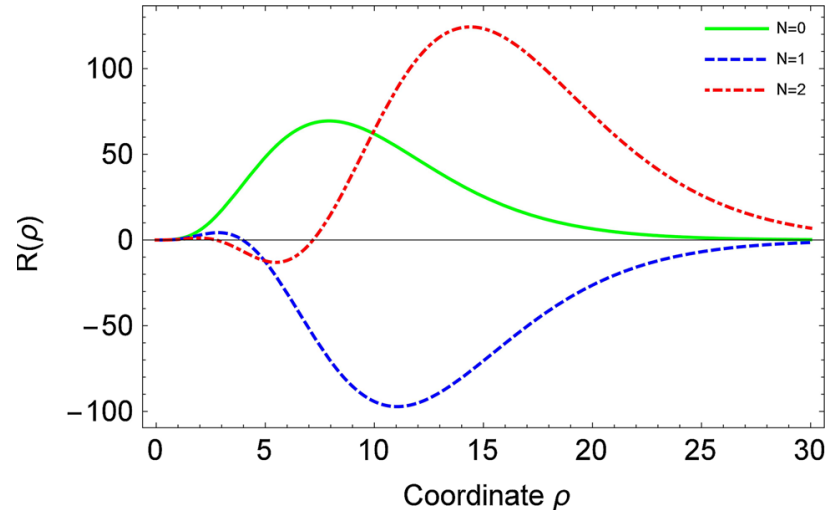

Fig. 1 The plots of the radial coordinate $R$ as the function of the variable $\rho$ displayed for three different values of $N$ with the parameters $\alpha=0.9, \omega=0.6, \eta=1$ and $l=1$

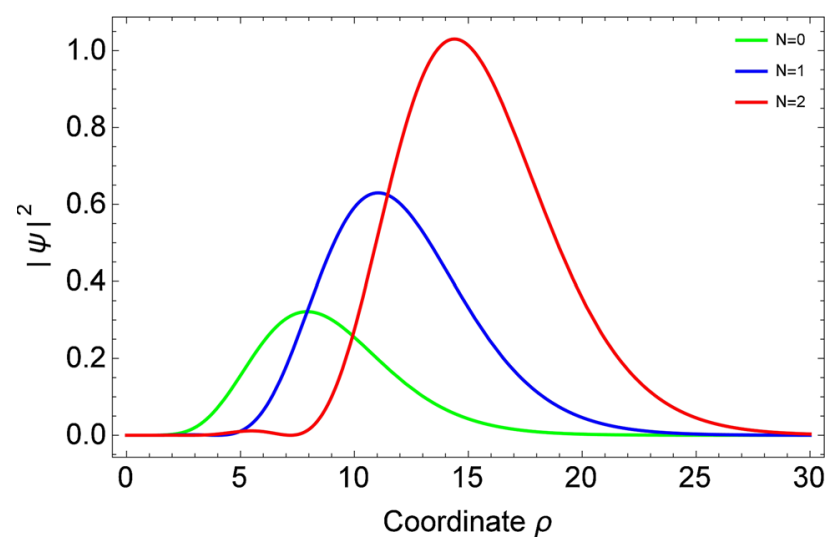

Fig. 2 The plots of $|\psi|^{2}$ as a function of the variable $\rho$ displayed for three different values of $N$ with the parameters $\alpha=0.9, \omega=0.6, \eta=1$ and $l=1$

Observing Eq. (20), we can see that the energy spectrum depends on $\alpha$, the deficit angle of the conical spacetime. The first and second terms are associated to the Coulomb-like potentials embedded in a cosmic string background and the third term is associated to the noninertial effect of rotating frames; it is a term due to Page-Werner et al. [44-47]. For $l=0$ or $\omega=0$ the discrete set of energies are symmetrical about $\varepsilon=0$; in this way, the presence of noninertial effects of rotating frames in spacetime breaks the symmetry of energy levels about $\varepsilon=0$ because $\varepsilon_{+}$, in general, is greater than $\varepsilon_{-}$.

From Eq. (20), it is possible to see that the energy depends on the constant $\alpha$, thus the presence of the topological defect, modifies the energy of the particle.

Figures 1 and 2 show that the radial solution $R(\rho)$ decreases with the coordinate $\rho$ and becomes negligible far away from the cosmic string as $\rho \rightarrow \infty$. For clarity, the plots of the energy spectrum $\varepsilon$ as a function of the variables $N$ and $l$ are shown in Figs. 3 and 4.

In the next section we will discuss an arbitrary relation $\omega \alpha$ for the Klein-Gordon oscillator where the shape of the potential is adequate for this purpose.

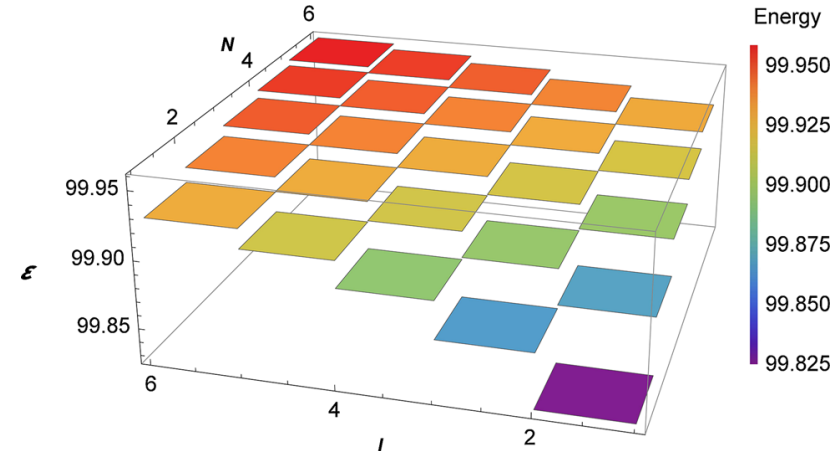

Fig. 3 The plots of particle energy spectrum $\varepsilon$ as the function of variables $N$ and $l$

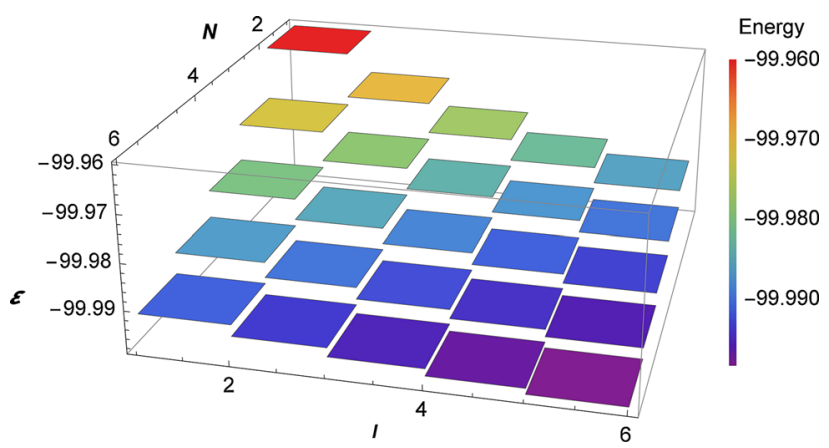

Fig. 4 The plots of negative energy spectrum $\varepsilon$ as a function of variables $N$ and $l$

\section{Klein-Gordon oscillator in the cosmic string spacetime}

Another system of interest that may be considered is the Klein-Gordon oscillator [48] in the background of the cosmic string spacetime. In recent years, several studies have addressed the Klein-Gordon oscillator in quantum systems [49-57]. It has a formulation similar to the vector potential in the previous section, so to study its solutions we will use the following change in momentum operator:

$p_{\mu} \rightarrow\left(p_{\mu}+i m \Omega X_{\mu}\right)$,

where $m$ is the particle mass at rest, $\Omega$ is the frequency of the oscillator and $X_{\mu}=(0, r, 0,0)$, with $r$ being the distance from the particle to the string. In this way, the Klein-Gordon equation becomes

$$
\left[-\frac{1}{\sqrt{-g}}\left(\partial_{\mu}+m \Omega X_{\mu}\right) g^{\mu \nu} \sqrt{-g}\left(\partial_{\nu}-m \Omega X_{\nu}\right)+(m+V)^{2}\right] \psi=0 .
$$

Taking $V=0$ in the above equation and by considering the line element (3), we obtain the following equation: 


$$
\begin{aligned}
& {\left[-\frac{\partial^{2}}{\partial t^{2}}+\frac{1}{r}\left(\frac{\partial}{\partial r}+m \Omega\right) r\left(\frac{\partial}{\partial r}-m \Omega\right)+\frac{\partial^{2}}{\partial z^{2}}\right.} \\
& \left.+\left(\frac{1-\alpha^{2} r^{2} \omega^{2}}{\alpha^{2} r^{2}}\right) \frac{\partial^{2}}{\partial \phi^{2}}+2 \omega \frac{\partial^{2}}{\partial t \partial \phi}-m^{2}\right] \psi=0 .
\end{aligned}
$$

Similar to the case of the Coulomb potential in the previous section, Eq. (23) is independent of $t, z$ and $\phi$, so it is appropriate to choose the ansatz

$\psi(t, r, z, \phi)=e^{-i \varepsilon t} e^{i l \phi} e^{i p_{z} z} R^{\prime}(r)$,

with $l=0, \pm 1, \pm 2, \pm 3$, and $\varepsilon$ being the energy of the particle. Substituting (24) into Eq. (23), we obtain the radial differential equation

$$
\begin{aligned}
& {\left[+\frac{1}{r}\left(\frac{\partial}{\partial r}+m \Omega\right) r\left(\frac{\partial}{\partial r}-m \Omega\right)+\right.} \\
& \left.\quad-\frac{l^{2}}{\alpha^{2} r^{2}}+(\varepsilon+\omega l)^{2}-p_{z}^{2}-m^{2}\right] R^{\prime}(r)=0
\end{aligned}
$$

At this stage, we can consider the substitution $R^{\prime}(r)=$ $R(r) / \sqrt{r}$ in Eq. (25) the result is

$$
\left[\frac{\mathrm{d}^{2}}{\mathrm{~d} r^{2}}-m^{2} \Omega^{2} r^{2}-\frac{\left(l^{2} / \alpha^{2}-1 / 4\right)}{r^{2}}+K^{2}\right] R(r)=0,
$$

with $K=\sqrt{(\varepsilon+\omega l)^{2}-p_{z}^{2}-m^{2}-2 m \Omega}$. This is the radial equation that describes the Klein-Gordon oscillator in the spacetime of a cosmic string. In order to obtain the solution to the above differential equation it is necessary to analyze its asymptotic behavior for $r \rightarrow 0$ and $r \rightarrow r_{0}$ where $r_{0} \equiv$ $1 / \omega \alpha$. In this way, a regular solution at the origin is obtained if the solution of Eq. (26) has the form

$R(r)=r^{\left|\frac{l}{\alpha}\right|+\frac{1}{2}} e^{-m \Omega r^{2} / 2} F(r)$.

Substituting the above expression in Eq. (26) and by introducing the new variable $\rho=m \Omega r^{2}$, we can rewrite the radial Eq. (26) in the form

$$
\begin{gathered}
\rho \frac{\mathrm{d}^{2} F(\rho)}{\mathrm{d} \rho^{2}}+\left(\left|\frac{l}{\alpha}\right|+1-\rho\right) \frac{\mathrm{d} F(\rho)}{\mathrm{d} \rho} \\
-\left(\frac{l}{2 \alpha}+\frac{1}{2}-\frac{K^{2}}{4 m \Omega}\right) F(\rho)=0 .
\end{gathered}
$$

The solution of Eq. (28) is given by the confluent hypergeometric function, which is denoted by

$$
F(\rho)={ }_{1} F_{1}(A, B ; \rho),
$$

where the parameters $A, B$ and $\rho$ are given by

$$
\begin{aligned}
& A=\frac{1}{2}\left|\frac{l}{\alpha}\right|+\frac{1}{2}-\frac{K^{2}}{4 m \Omega}, \\
& B=\left|\frac{l}{\alpha}\right|+1, \\
& \rho=m \Omega r^{2} .
\end{aligned}
$$

\subsection{Limit $\alpha \omega \ll 1(1 / \alpha \omega \rightarrow \infty)$}

Following the discussions of Sect. 3, we proceed now to finding the eigenfunction for this problem.

Considering again the limit $\alpha \omega \ll 1$, we have a change in the boundary condition on the radial coordinate, i.e., when $\alpha \omega \ll 1$ the radial coordinate tends to infinity at $r=1 / \omega \alpha$. Consequently the hypergeometric function must be a polynomial function of degree $N$, and the parameter $A=\frac{1}{2}\left|\frac{l}{\alpha}\right|+\frac{1}{2}-\frac{K^{2}}{4 m \Omega}$ must be a negative integer. This condition implies that

$\frac{1}{2}\left|\frac{l}{\alpha}\right|+\frac{1}{2}-\frac{K^{2}}{4 m \Omega}=-N$

and by the use of the definition of

$K=\sqrt{(\varepsilon+\omega l)^{2}-p_{z}^{2}-m^{2}-2 m \Omega}$,

we finally obtain the set of energies

$$
\begin{gathered}
\varepsilon= \pm \sqrt{2 m \Omega\left(2 n^{\prime}+\left|\frac{l}{\alpha}\right|\right)+m^{2}+p_{z}^{2}}-\omega|l| \\
n^{\prime} \equiv N+1=1,2,3, \ldots
\end{gathered}
$$

We can see that the energy spectrum associated with the Klein-Gordon oscillator in the conical spacetime depends on $\alpha$, that is, the deficit angle of the conical spacetime. It increases the energy of the system if $\alpha<1$. It is easy to see that for $l=0$ or $\omega=0$ the energy is symmetrical about $\varepsilon=$ 0 . In this way, the rotating frame breaks the symmetry of the energy about $\varepsilon=0$. The first term in Eq. (35) is associated to the Klein-Gordon oscillator embedded in a conical space and the second one is associated with the noninertial effect, which in turn is a coupling between the angular quantum number and the angular velocity of the rotating frame. As may be seen in Fig. 5, the radial eigenfunction becomes negligible far away from the string as $\rho \rightarrow \infty$. Figure 6 presents $|\psi|^{2}$ as a function of the variable $\rho$ for three different values of $n^{\prime}$. The energy spectrum as a function of the variables $n^{\prime}$ and $l$ are shown in the plots of Figs. 7 and 8. We note that the result obtained in Eq. (35) is similar to the one reported in [2] for scalar bosons described by the Duffin-Kemmer-Petiau (DKP) formalism. 


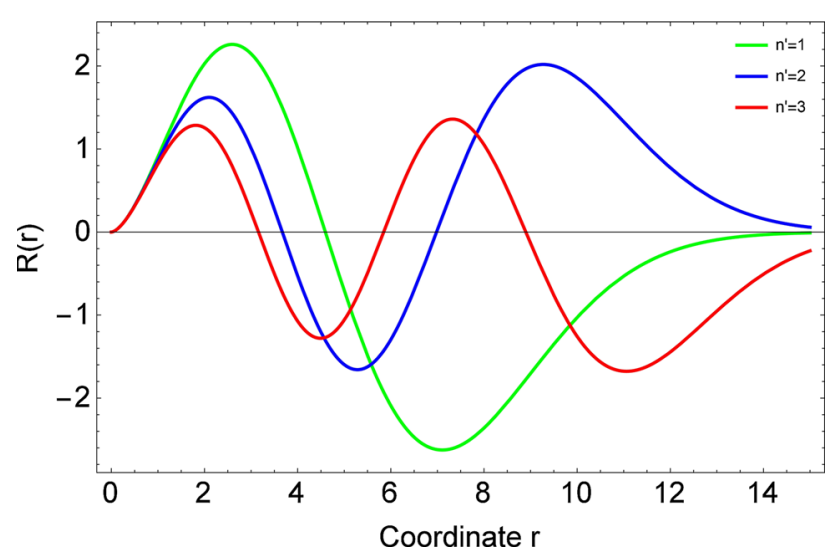

Fig. 5 The plots of radial coordinate $R$ as the function of variable $r$ displayed for three different $n^{\prime}$ with the parameters $\alpha=0.9, \omega=0.6$, $\Omega=0.1, m=1$ and $l=1$

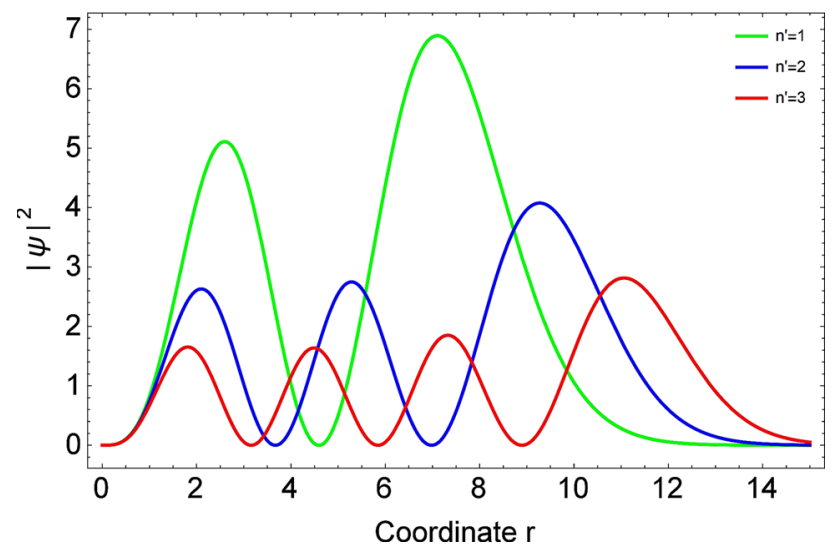

Fig. 6 The plots of $|\psi|^{2}$ as functions of the variable $\rho$ displayed for three different values of $n^{\prime}$ with the parameters $\alpha=0.9, \omega=0.6$, $\Omega=0.1, m=1$ and $l=1$

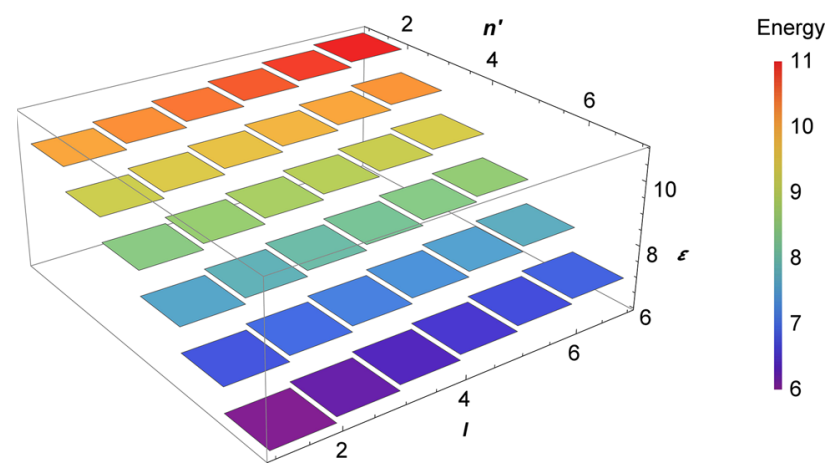

Fig. 7 The plots the of particle energy spectrum $\varepsilon$ as function of the variables $n^{\prime}$ and $l$

\subsection{Arbitrary $\omega \alpha$}

Now let us study an arbitrary relation between the parameters $\alpha$ and $\omega$. In this case we discuss the behavior of the KleinGordon oscillator without assuming the limit $\alpha \omega \ll 1$. The physical condition implies that the wave function vanishes at $r_{0}=1 / \alpha \omega$, i.e.,

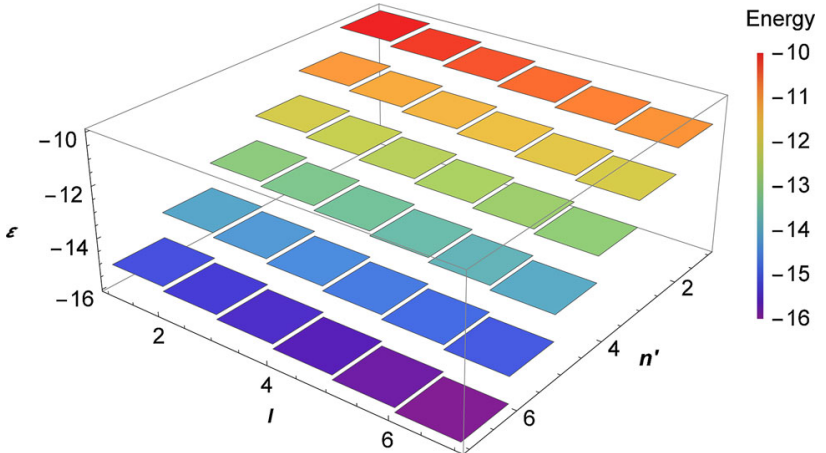

Fig. 8 The plots of the negative energy spectrum $\varepsilon$ as a function of the variables $n^{\prime}$ and $l$

${ }_{1} F_{1}\left(A, B ; \rho_{0}=m \Omega r_{0}^{2}\right)=0$.

If one assumes that $m \Omega \ll 1$, the parameter $A$ of the hypergeometric function can be considered large and the parameters $B$ and $r_{0}$ remain fixed. Thus, we can use these results to expand the hypergeometric function in the form $[58,59]$

$$
\begin{aligned}
{ }_{1} F_{1}\left(A, B ; \rho_{0}\right) \approx & \frac{\Gamma(B)}{\sqrt{\pi}} e^{\frac{\rho_{0}}{2}\left(\frac{B \rho_{0}}{2}-A \rho_{0}\right) \frac{1-B}{2}} \\
& \times \cos \left(\sqrt{2 B \rho_{0}-4 A \rho_{0}}-\frac{B \pi}{2}+\frac{\pi}{4}\right) ;
\end{aligned}
$$

here $\Gamma(B)$ is the gamma function. By considering the condition (36) and Eq. (37), we finally obtain the set of energies for an arbitrary relation between the parameters $\alpha$ and $\omega$,

$\varepsilon \approx \sqrt{p_{z}^{2}+m^{2}+2 m \Omega+\frac{1}{r_{0}^{2}}\left(\frac{l \pi}{2 \alpha}+\frac{3 \pi}{4}+n \pi\right)^{2}}-\omega l$

where $n=0,1,2, \ldots$ is the radial quantum number of solution. Then Eq. (38) corresponds to the set of energies for an arbitrary relation between $\alpha$ and $\omega$ where the noninertial effects play o hole of an hard-wall confining potential [59]. The first term of Eq. (38) is associated to the Klein-Gordon oscillator embedded in a cosmic string background and the second term is associated to the noninertial effect of rotating frames, which in turn is a Sagnac-type effect.

\section{Conclusions}

In this work, we have determined the Klein-Gordon equation in the presence of a vector and a scalar potential and examined the Klein-Gordon wave equation in the presence of a Klein-Gordon oscillator in a cosmic string spacetime. 
Despite the complexity of the studied systems, we obtained compact expressions for the energy spectrum and for the particles wave functions. It has been shown that the potentials studied allow the formation of bound states and the energy spectrum associated with the Klein-Gordon equation in a cosmic string space depends on the deficit angle $\alpha$. This fact shows that the topological defect modifies the energy of physical systems.

An important result that we have shown is that the noninertial effect restricts the region of the spacetime where the particle can be observed and beyond that, it shifts the energy levels. This feature reveals the existence of a coupling between the angular quantum number and the angular velocity of the rotating frame.

We have shown that the Klein-Gordon equation presents two different classes of solutions that depend on the value of the $\alpha$ and $\omega$. In the first case the limit $\alpha \omega \ll 1$ is assumed, that is, the slow rotation regime, and as a second case, an arbitrary relation for $\alpha \omega$ is considered. For both classes of solutions, we have found the energy spectrum and the eigenfunctions and we have shown that the discrete set of energies in general is composed of two contributions. The first term is associated to the external potential embedded in a cosmic string background and the second one is associated to noninertial effects. With these results it is possible to have an idea as regards the general aspects of the quantum dynamics of scalar bosons inside a cosmic string background.

Therefore, in this paper, we have shown some results for quantum systems where general relativistic effects are taken into account, which in addition with the previous results $[25,38]$ present many interesting effects. This is a fundamental subject in physics, and the connections between these theories are not well understood.

Acknowledgements This work was supported in part by means of funds provided by CAPES.

Open Access This article is distributed under the terms of the Creative Commons Attribution 4.0 International License (http://creativecomm ons.org/licenses/by/4.0/), which permits unrestricted use, distribution, and reproduction in any medium, provided you give appropriate credit to the original author(s) and the source, provide a link to the Creative Commons license, and indicate if changes were made.

Funded by SCOAP ${ }^{3}$.

\section{References}

1. L.B. Castro, Eur. Phys. J. C 76, 1 (2016)

2. L.B. Castro, Eur. Phys. J. C 75, 1 (2015)

3. R.N.C. Filho, J.P. Braga, J.H. Lira, J.S.A. Jr, Phys. Lett. A 755, 367 (2016)

4. L. Parker, Phys. Rev. Lett. 44, 1559 (1980)

5. L. Parker, Phys. Rev. D 22, 1922 (1980)

6. L. Parker, Phys. Rev. D 24, 535 (1981)

7. L. Parker, Gen. Relat. Gravit. 13, 307 (1981)
8. L. Parker, L. Pimentel, Phys. Rev. D 25, 3180 (1982)

9. G. Marques, V. Bezerra, Phys. Rev. D 66, 105011 (2002)

10. C.C. Barros, Eur. Phys. J. C 42, 119 (2005)

11. C. Barros, Eur. Phys. J. C 45, 421 (2006)

12. S. Chandrasekhar, Proc. R. Soc. Lond. Ser. A Math. Phys. Sci. 349, 571 (1976)

13. J.M. Cohen, R.T. Powers, Commun. Math. Phys. 86, 69 (1982)

14. D.R. Brill, J.A. Wheeler, Rev. Mod. Phys. 29, 465 (1957)

15. A.D. Linde, Rep. Progr. Phys. 42, 389 (1979)

16. A. Vilenkin, Phys. Rev. D 24, 2082 (1981)

17. A. Vilenkin, Phys. Rev. D 23, 852 (1981)

18. R. Poltis, D. Stojkovic, Phys. Rev. Lett. 105(161301), 15 (2010)

19. W.A. Hiscock, Phys. Rev. D 31, 3288 (1985)

20. M. Aryal, L.H. Ford, A. Vilenkin, Phys. Rev. D 34, 2263 (1986)

21. M.G. Germano, V.B. Bezerra, E.R.B. de Mello, Class. Quant. Gravit. 13, 2663 (1996)

22. S. Ölmez, V. Mandic, X. Siemens, Phys. Rev. D 81, 104028 (2010)

23. B.P. Abbott, R. Abbott, M. Abbott, Y. Zhang, C. Zhao, M. Zhou, Z. Zhou, X.J. Zhu, M.E. Zucker, S.E. Zuraw, J. Zweizig, LIGO Scientific Collaboration and Virgo Collaboration, Phys. Rev. Lett. 116, 061102 (2016)

24. J. Carvalho, A. de M. Carvalho, C. Furtado, Eur. Phys. J. C 74 (2014). https://doi.org/10.1140/epjc/s10052-014-2935-y

25. L.C.N. Santos, C.C. Barros, Eur. Phys. J. C 76, 560 (2016)

26. L. C. N. Santos, C. C. Barros (2017). arXiv:1704.00408

27. Ø. Grøn, Am. J. Phys. 43, 869 (1975)

28. Ø. Grøn, Int. J. Theor. Phys. 16, 603 (1977)

29. K. Konno, R. Takahashi, Phys. Rev. D 85, 061502 (2012). arXiv:1201.5188 [gr-qc]

30. M. Hosseinpour, H. Hassanabadi, Eur. Phys. J. Plus 130, 236 (2015). arXiv:1505.00096 [hep-th]

31. H.F. Mota, K. Bakke, Phys. Rev. D 89, 027702 (2014). arXiv: 1401.3728 [hep-th]

32. D. Chowdhury, B. Basu, Ann. Phys. 339, 358 (2013). arXiv:1309.1376 [cond-mat.meshall]

33. M. Dvornikov, Mod. Phys. Lett. A 30, 1530017 (2015). arXiv:1503.01431 [hep-ph]

34. V.E. Ambrus, E. Winstanley, Phys. Rev. D 93, 104014 (2016). arXiv: 1512.05239 [hep-th]

35. K. Bakke, Phys. Lett. A 374, 4642 (2010)

36. K. Bakke, Mod. Phys. Lett. B 27, 1350018 (2013)

37. B. Mashhoon, Phys. Rev. Lett. 61, 2639 (1988)

38. L.C.N. Santos, C.C. Barros, Eur. Phys. J. C 77, 186 (2017)

39. K. Bakke, C. Furtado, Phys. Rev. D 80, 024033 (2009)

40. K. Bakke, C. Furtado, Phys. Rev. D 82, 084025 (2010)

41. N. Birrell, P. Davies, Quantum Fields in Curved Space, Cambridge Monographs on Mathematical Physics (Cambridge University Press, Cambridge, 1984)

42. A.L. Cavalcanti de Oliveira, E.R. Bezerra de Mello, Class. Quant. Gravit. 23, 5249 (2006). arXiv:hep-th/0603036 [hep-th]

43. E.R. Figueiredo Medeiros, Eur. Phys. J. C72, 2051 (2012). arXiv:1108.3786 [hep-th]

44. F. Hehl, W.-T. Ni, Phys. Rev. D 42, 2045 (1990)

45. M. G. Sagnac, C. R. Acad. Sci. (Paris) 157, 708 (1913)

46. E. Post, Rev. Mod. Phys. 39, 475 (1967)

47. M. G. Sagnac, C. R. Acad. Sci. (Paris) 157, 1410 (1913)

48. S. Bruce, P. Minning, Il Nuovo Cimento A 106, 711 (1993)

49. J. Carvalho, A.M. de M. Carvalho, E. Cavalcante, C. Furtado, Eur. Phys. J. C76, 365 (2016). arXiv:1603.06292 [hep-th]

50. Y.-J. Xiao, Z.-W. Long, S.-H. Cai, Int. J. Theor. Phys. 50, 3105 (2011)

51. W. Jian-Hua, L. Kang, D. Sayipjamal, Chin. Phys. C 32, 803 (2008)

52. Q. Wen-Chao, Chin. Phys. 12, 1054 (2003)

53. K. Bakke, C. Furtado, Ann. Phys. 355, 48 (2015). arXiv: 1411.6988 [quant-ph] 
54. R.V. Maluf, Int. J. Mod. Phys. A 26, 4991 (2011). arXiv:1101.2801 [hep-th]

55. R.L.L. Vitria, C. Furtado, K. Bakke, Ann. Phys. 370, 128 (2016). arXiv:1511.05072 [quant-ph]

56. M.-L. Liang, R.-L. Yang, Int. J. Mod. Phys. A 27, 1250047 (2012)

57. N.A. Rao, B. Kagali, Phys. Scripta 77, 015003 (2007)
58. M. Abramowitz, I. Stegun, Handbook of Mathematical Functions: With Formulas, Graphs, and Mathematical Tables, Applied Mathematics Series (Dover Publications, New York, 1964)

59. K. Bakke, Gen. Relat. Gravit. 45, 1847 (2013). arXiv:1307.2847 [quant-ph] 